Mon. Not. R. Astron. Soc. 000,1-?? (2002) Printed 1 March $2022 \quad$ (MN ঝّT $\mathrm{E}$ X style file v2.2)

\title{
Thermal generation of the magnetic field in the surface layers of massive stars
}

\author{
V. Urpin ${ }^{1,2)}$ \\ ${ }^{1}$ INAF, Osservatorio Astrofisico di Catania, Via S.Sofia 78, 95123 Catania, Italy \\ 2) A.F.Ioffe Institute of Physics and Technology, 194021 St. Petersburg, Russia
}

today

\begin{abstract}
A new magnetic field-generation mechanism based on the Nernst effect is considered in hot massive stars. This mechanism can operate in the upper atmospheres of $\mathrm{O}$ and $\mathrm{B}$ stars where departures from the LTE form a region with the inverse temperature gradient.
\end{abstract}

Key words: stars: massive - stars: magnetic field - stars: atmospheres - stars: instabilities

\section{INTRODUCTION}

" The investigations of stellar magnetism and its influence on the stellar surfaces is a quickly maturing research area. Recently, a number of studies have been carried out on a detection of the magnetic field in massive B- and O-type stars (see, e.g., Schöller et al. 2016, ' Hubrig et al. 2016). While in a number of B stars, the magnetic field was detected already several decades ago, the existence of magnetic ' fields in $\mathrm{O}$ stars has been proven only recently. The direct field mea, surements are difficult in such stars since only a few spectral lines - are available for this. Besides, the average magnetic field of $\mathrm{O}$ stars turns out to be $\sim 8-9$ times weaker than the field of B and A stars (Kholtygin et al. 2015).

The origin of magnetic fields in massive stars is still poorly understood. Upper main-sequence stars with $M>2 M_{\odot}$ have no convective envelopes and it seems that dynamo should not be efficient in OB stars. Therefore, some authors argue that the magnetic field of these stars is fossil or generated by some exotic mechanisms (like, e.g., interaction in stellar mergers or in the course of a mass transfer). However, the role of dynamo can be underestimated. For instance, Cantiello \& Braithwaite (2011) considered the subsurface mechanism, assuming that dynamo generates the magnetic field in the Fe convection zone. They found that the field generated in this zone could emerge at the surface via magnetic buoyancy and be widespread. Also, it has been shown that a significant fraction of OB stars may suffer merger or mass-transfer events in their evolution and the field can be generated through such processes (e.g., Sana et al. 2012, Wickramasinghe et al. 2015).

Dynamo, however, is not the only mechanism that amplifies the magnetic field in astrophysical bodies. Sometimes, the electric currents supporting the magnetic field can be related to other mechanisms. For instance, the Biermann battery is an example of such non-dynamo mechanism (see, e.g., Biermann 1950, Kemp 1982, Mestel \& Moss 1983) that operates in plasma if gradients of the pressure and number density of electrons are non-parallel. Several astrophysical applications of this process have been discussed that range from stellar magnetic fields to seed magnetic fields on galactic scales. For example, this process can generate a seed magnetic field around first stars (see, e.g., Ando et al. 2010, Doi \& Susa 2011). Note that a similar mechanism (often called the crosshelicity effect) operates in turbulent plasma (Yoshizava 1990, Brandenburg \& Urpin 1998). In this case, the role of the pressure gradient is played by the gradient of turbulent stresses.

In this paper, we consider the mechanism of a magnetic field generation that operates in plasma with a non-uniform temperature. This mechanism is caused by the so-called thermomagnetic instability and involves a range of processes operating in hot plasmas. The thermomagnetic processes transform a fraction of the thermal flux into the energy of magnetic fields. Such possibility first was considered with regards to experiments in the laser plasma (see, e.g., Tidman \& Shanny 1974, Bol'shov et al. 1974, Dolginov \& Urpin 1979). In this scenario, a feedback acts between the Nernst effect and the Righi-Leduc heat flow and is determined entirely by transport processes and neither hydrodynamic motions nor density gradients are required. In the laser plasma, the thermomagnetic processes lead to the instability that generates strong magnetic fields ( $\left.\sim 10^{6}-10^{7} \mathrm{G}\right)$ on a short timescale (see, e.g., Haines 1981, Andrushchenko \& Pavlenko 2004). The impact of both density gradients and hydrodynamic motions on the thermomagnetic instability is considered by Bissell et al. 2010, 2012. Hydrodynamic motion is found to produce a limited effect on the growth rate, whereas the influence of density gradients can be more essential because they produce an additional source term. In un-magnetised plasmas, it is widely believed that this instability may be driven by two mechanisms which are determined by i) non-parallel gradients of the temperature and electron number density and ii) the Nernst advection that can lead to exponential compression of the magnetic perturbations (see Bissell 2015 for more detail). Note that the Nernst advection can contribute to the field generation if temperature and density gradients are parallel and if this condition fails (Brownell 1979, Hirao \& Ogasawara 1981).

Under astrophysical conditions, this instability has been considered in the context of the early evolution of neutron stars where it generates strong magnetic fields in the surface layers (see Urpin 
et al. 1986). The evolution of thermally generated magnetic fields can account for a number of qualitative features observed in pulsars (such as fast decay at the early evolution and slow decay during the later evolution; see Urpin \& van Riper 1993, Urpin et al. 1994). Note that thermomagnetic processes can occur in non-magnetized as well as magnetized plasma, and motions caused by these processes influence transport properties of plasma. The mechanism for such instability in magnetized plasmas is well studied. Generally, feedback between the Nernst effect and the Righi-Leduc heat flow can be caused by transport processes and neither hydrodynamic motions nor density gradients are required. The instability yields propagating magnetothermal waves whose direction depends on the magnitude of the Hall parameter. For instance, these processes are important in hot accretion discs (Montani et al. 2013, Urpin \& Rüdiger 2005, Franko et al. 2014) where the thermomagnetic phenomena are accompanied by the magnetorotational ones and contribute to the angular momentum transport.

In this paper, we consider the thermomagnetic processes that operate in the surface layers of massive stars. Plasma in the upper atmosphere of OB stars is hot and its density is relatively low. Therefore, thermomagnetic phenomena can lead to an efficient generation of the magnetic field. We argue that characteristics of the magnetic field generated by this mechanism in massive stars are in qualitative agreement with observational data.

\section{BASIC EQUATIONS}

Consider the thermomagnetic instability in the upper atmosphere by making use of a plane-parallel geometry with $(x, y, z)$ being the Cartesian coordinates. We assume that within the layer between $z=0$ (bottom) and $z=a$ (top), the temperature gradient is directed in a positive or negative $z$-direction. The generalized Ohm's law in fully ionised plasma reads (see, e.g., Braginskii 1965)

$\vec{E}=-\frac{\vec{v}}{c} \times \vec{B}-\frac{\vec{B} \times \vec{j}}{e n_{e}}-\frac{\nabla p_{e}}{e n_{e}}+\frac{\hat{\alpha} \cdot \vec{j}}{\left(e n_{e}\right)^{2}}-\frac{\hat{\beta} \cdot \nabla T}{e n_{e}}$,

where $\vec{E}, \vec{B}$, and $\vec{j}$ are the electric and magnetic fields and the electric current, $\vec{v}$ is the velocity, $n_{e}$ and $p_{e}$ are the number density and pressure of electrons, respectively; $T$ is the temperature; $e$ is the electron charge, $\hat{\alpha}$ and $\hat{\beta}$ are tensors that characterize the rate of dissipation of electric currents and the efficiency of thermomagnetic phenomena. The tensor productions in Eq. (1) read

$$
\begin{array}{r}
\hat{\alpha} \cdot \vec{j}=\alpha_{\|} j_{\|}+\alpha_{\perp} j_{\perp}-\alpha_{\wedge} \vec{b} \times \vec{j}, \\
\hat{\beta} \cdot \nabla T=\beta_{\|} \nabla_{\|} T+\beta_{\perp} \nabla_{\perp} T+\beta_{\wedge} \vec{b} \times \nabla T,
\end{array}
$$

where $\vec{b}=\vec{B} / B$; the subscripts $\|, \perp$, and $\wedge$ mark the components parallel and perpendicular to the magnetic field and the so-called Hall component. The components of $\hat{\alpha}$ and $\hat{\beta}$ depend on the parameters of plasma and its chemical composition. For one component fully ionized plasma, these coefficients have been calculated by Braginskii (1965). The kinetic coefficients in a multicomponent plasma have been considered by Urpin (1981).

Combining the Faraday's law $\partial \vec{B} / \partial t=-c \nabla \times \vec{E}$ with Eq. (1), we obtain the induction equation

$$
\begin{aligned}
\frac{\partial \vec{B}}{\partial t}- & \nabla \times(\vec{v} \times \vec{B})-\frac{c}{e} \nabla \times\left(\frac{\vec{B} \times \vec{j}}{n_{e}}\right)-\frac{c}{e} \nabla \times\left(\frac{\nabla p_{e}}{n_{e}}\right) \\
+ & \frac{c}{e^{2}} \nabla \times\left(\frac{1}{n_{e}^{2}} \hat{\alpha} \cdot \vec{j}\right)-\frac{c}{e} \nabla \times\left(\frac{1}{n_{e}} \hat{\beta} \cdot \nabla T\right)=0 .
\end{aligned}
$$

Consider a particular type of waves governed by this equation. We use the standard linear approach that is valid for waves with small amplitude and we represent all quantities as a sum of the unperturbed quantity and a small disturbance that will be marked by a subscript 1 . The disturbances are governed by linearized MHD equations while they are small compared to the unperturbed quantities. We assume that unperturbed velocity and magnetic field are vanishing. Linearizing tensors $\hat{\alpha}$ and $\hat{\beta}$, we take into account that all terms $\propto B^{2}$ should be neglected because there is no magnetic field in the basic state. Then, we have for fully ionized hydrogen plasma

$$
\begin{array}{r}
\alpha_{\|} \approx \alpha_{\perp}=0.51 \frac{m_{e} n_{e}}{\tau_{e}}, \quad \beta_{\|} \approx \beta_{\perp}=0.71 n_{e} k_{B}, \\
\beta_{\wedge}=0.81 \frac{e n_{e} k_{B} \tau_{e}}{m_{e} c} B_{1},
\end{array}
$$

where $k_{B}$ is the Boltzmann constant and $\tau_{e}$ is the electron relaxation time (Braginskii 1965). In fully ionized hydrogen plasma, the relaxation time of electrons is given by $\tau_{e}=$ $3 \sqrt{m_{e}}\left(k_{B} T\right)^{3 / 2} / 4 \sqrt{2 \pi} e^{4} n_{e} \Lambda$ where $\Lambda$ is the Coulomb logarithm (see, e.g., Spitzer 1998). The coefficient $\alpha_{\wedge}$ is proportional to $B_{1}$ but it should be multiplyed in Eq.(3) by the electrc current that is $\vec{j}_{1}=(c / 4 \pi) \nabla \times \vec{B}_{1}$ and, hence, this term is non-linear in a small disturbance $B_{1}$ and must be neglected. Therefore, the linearized induction equation reads

$$
\begin{array}{r}
\frac{\partial \vec{B}_{1}}{\partial t}=-\frac{c^{2}}{4 \pi} \nabla \times\left(\frac{1}{\sigma} \nabla \times \vec{B}_{1}\right)+ \\
\frac{c}{e} \nabla \times\left(\frac{\nabla p_{e 1}}{n_{e}}-\frac{n_{e 1} \nabla p_{e}}{n_{e}^{2}}\right)-0.81 \frac{k_{B}}{m_{e}} \nabla \times\left(\tau_{e} \nabla T \times \vec{B}_{1}\right),
\end{array}
$$

where $\sigma=e^{2} n_{e} \tau_{e} / 0.51 m_{e}$ is the conductivity along the magnetic field. The first term on the r.h.s. of Eq.(5) describes the standart Ohmic dissipation that always operates in plasma, the second term describes magnetic field generation by the Biermann battery $\left(\propto \nabla T \times \nabla n_{e}\right)$, and the third term corresponds to Nernst advection of the magnetic field lines.

Eq. (5) should be complemented by the heat balance, momentum, and continuity equations. The heat equation reads

$\rho c_{p} \frac{d T}{d t}-\frac{d p}{d t}=-\nabla \cdot \overrightarrow{q_{e}}-\nabla \cdot \vec{Q}+G-\Lambda$,

where $\rho$ and $p$ are the density and total pressure, respectively; $c_{p}$ is the specific heat for $p=$ const; $G$ and $\Lambda$ are the heating and cooling rates; $\overrightarrow{q_{e}}=-\hat{\kappa_{e}} \cdot \nabla T$ is the heat flux transported by electrons with $\hat{\kappa_{e}}$ being the tensor of electron thermal conductivity; $\vec{Q}$ describes the radiative heat flux; $d / d t=\partial / \partial t+(\vec{v} \cdot \nabla)$. The tensor of electron thermal conductivity is given by the standard expression (see, e.g., Braginski 1965). The radiative heat flux, $\vec{Q}$, has a simple form in optically thick layers where $\vec{Q}=-\kappa_{r} \nabla T$ with $\kappa_{r}$ being the radiation thermal conductivity. Note that this simple expression does not describe the radiative flux in a region with the optical depth $\leqslant 1$.

In the optically thin region, the radiative flux has a more complicated shape and should be considered by making use of the radiative transfer equation. The expression for $\vec{Q}$ in Eq. (6) is determined obviously by the thermodynamic parameters of plasma. Various approximate approaches have been suggested to describe this quantity in optically thin regions. For our purposes, we can choose the approach used by Wang (1966) and Ojha (1987). Following Wang (1966), the quantity $\vec{Q}$ can be represented as a power low function of the density and temperature,

$\nabla \cdot \vec{Q} \approx a c W_{0} \rho^{1+\zeta} T^{4+\iota}$ 
with $W_{0}, \zeta$, and $\iota$ being constants determined by fitting with opacity data. Note that the results of our study are not sensitive to a particular shape of the dependence of $\vec{Q}$ on $\rho$ and $T$. The only important point is that $\vec{Q}$ is weakly dependent on $B$ but this is certainly the case in stellar atmospheres where the magnetic field is of the order of $10^{2}-10^{3} \mathrm{G}$ because radiative opacities are not sensitive to $B$ for such fields. The absorption coefficient becomes to be dependent on $B$ only in a much stronger field $\sim 10^{8}-10^{9} \mathrm{G}$. If $\vec{Q}$ depends weakly on $B$ then our conclusions are the same for any dependence of $\vec{Q}$ on $\rho$ and $T$.

The thermomagnetic effects are determined by the temperature gradient rather than the electron heat-flux. In stellar atmospheres, the heat transport by electrons is much less efficient than the radiative transport and $Q \gg q_{e}$. For instance, it can be estimated that $Q$ is more than 15 orders of magnitude greater than $q_{e}$ in the layers with the optical depth $\sim 1$. In the region with a smaller optical depth, electron transport becomes a bit more efficient but still much less important than the radiative one. Therefore, $q_{e}$ plays no essential role in thermal balance and the first term on the r.h.s. of Eq. (6) can be neglected. This is a qualitative difference to the thermomagnetic instability in laser-produced plasma where the electron thermal conductivity is the dominating mechanism of heat transport (see, e.g., Tidman \& Shanny 1974, Bissell 2015, Bissell et al. 2012, Brownell 1979, and Hirao \& Ogasawara 1981). As far as the contribution of small terms proportional to the electron thermal conductivity is concerned, it is easy to estimate that they give a correction to the dispersion equation of the order of a ratio (electron thermal conductivity)/(radiative thermal conductivity) that is extremely small in stellar condition.

The momentum and continuity equations read

$$
\begin{array}{r}
\frac{d \vec{v}}{d t}=-\frac{\nabla p}{\rho}+\vec{g}+\frac{1}{4 \pi}(\nabla \times \vec{B}) \times \vec{B}, \\
\frac{\partial \rho}{\partial t}+\nabla \cdot(\rho \vec{v})=0,
\end{array}
$$

where $\vec{g}$ is gravity. In the case $\vec{q}_{e} \neq 0$, linearization of Eq.(6) and Eqs.(7)-(8) leads to the set of equations containing disturbances of $T_{1}, \rho_{1}, p_{1}, \vec{B}_{1}$, and $\vec{v}_{1}$. However, this set does not contain $\vec{B}_{1}$ if $q_{e}$ is neglected. Indeed, if $q_{e}$ is negligible, the only term in Eqs. (6) and (7)-(8) that depends on $\vec{B}_{1}$ is the Lorentz force in the momentum equation (7). Linearizing the Lorentz force, we have $[(\nabla \times \vec{B}) \times$ $\vec{B}]_{1}=\left(\nabla \times \vec{B}_{1}\right) \times \vec{B}+(\nabla \times \vec{B}) \times \vec{B}_{1}=0$ since $\vec{B}=0$ in the basic state. Therefore, Eqs.(6), (7) and (8) can be decoupled from Eq.(5) and these equations have their own set of eigenmodes and eigenvalues. On the other hand, the particular type of eigenmodes caused by the thermomagnetic effects can be described by Eq.(5) alone if one supposes that $T_{1}, \rho_{1}, p_{1}$, and $\vec{v}_{1}$ are vanishing. Then, only disturbances of $\vec{B}_{1}$ are non-vanishing in this particular mode that can be called thermomagnetic. In the case $\vec{q}_{e} \approx 0$, the equation governing this mode reads

$$
\frac{\partial \vec{B}_{1}}{\partial t}=-0.81 \frac{k_{B}}{m_{e}} \nabla \times\left(\tau_{e} \nabla T \times \vec{B}_{1}\right)-\frac{c^{2}}{4 \pi} \nabla \times\left(\frac{1}{\sigma} \nabla \times \vec{B}_{1}\right) .
$$

The thermomagnetic modes exist if the temperature is non-uniform. Comparing the first and second terms on the r.h.s. of Eq.(10) and assuming that the lendth-scales of perturbations and unperturbed quantities are of the same order, we obtain that thermomagnetic effects yield a stronger influence than ohmic dissipation if

$\varepsilon \equiv \frac{c_{e}^{2}}{c^{2}} \omega_{p}^{2} \tau_{e}^{2} \gg 1$ where $c_{e}=\sqrt{k_{B} T / m_{e}}$ is the thermal velocity of electrons and $\omega_{p}=\sqrt{4 \pi e^{2} n_{e} / m_{e}}$ the plasma frequency. Then, Eq. (11) yields

$\varepsilon \approx 36 T_{4}^{4} / n_{13} \Lambda^{2} \gg 1$,

where $n_{13}=n / 10^{13} \mathrm{~cm}^{-3}$ and $T_{4}=T / 10^{4} K$. If the wavelength of perturbations is much shorter than the unperturbed length-scale, then the Nernst effect is especially effective in the limit of longwavelength perturbations when dissipation is minimized (see Hirao \& Ogasawara (1981) and Bissell (2015)).

We consider Eq.(9) in the case $\varepsilon \gg 1$ when the thermomagnetic effects are important. Note that thermomagnetic modes have a simple form (9) only in a linear approximation when one does not take into account the Hall parameter and Lorenz force.

\section{THERMOMAGNETIC INSTABILITY}

It is seen from Eq.(14) that thermomagnetic effects can influence only the component of $\vec{B}_{1}$ perpendicular to $\nabla T$. It is convenient to choose the $y$-axis parallel to $\vec{B}_{1}$. If the basic state is quasistationary, $\vec{B}_{1}$ depends on $t$ and $x$ as $\propto \exp \left(\gamma t-i k_{x} x\right)$ where $\gamma$ is the growth rate and $k_{x}$ is the wavevector in the $x$-direction. The dependence on $z$ should be determined from Eq.(14). Under these assumptions, the equation governing the magnetic field reads

$\eta_{m} B_{1 y}^{\prime \prime}+A B_{1 y}^{\prime}+D B_{1 y}=0$,

where $\eta_{m}=c^{2} / 4 \pi \sigma$ is the magnetic diffusivity and

$$
\begin{gathered}
A=0.81 \frac{k_{B}}{m_{e}} \tau_{e} \frac{d T}{d z}-\eta_{m} \frac{d \ln \sigma}{d z}, \\
D=D_{0}-\eta_{m} k_{x}^{2}-\gamma, \quad D_{0}=0.81 \frac{k_{B}}{m_{e}} \frac{d}{d z}\left(\tau_{e} \frac{d T}{d z}\right) ;
\end{gathered}
$$

the prime denotes $d / d z$. The ratio of the first and second terms on the r.h.s. of the expression for $A$ is of the order of $\varepsilon$ and, therefore, the second term $(\propto(d \ln \sigma / d z))$ can be neglected in the region where thermomagnetic effects play a dominant role.

To illustrate the main qualitative features of thermomagnetic modes, we consider the behaviour of disturbances with a wavelength in the $z$-direction that is much shorter than the characteristic lengthscales of unperturbed quantities. Then the solution of Eq.(13) is $\propto \exp \left[i \int q(z) d z\right]$ and we have

$B_{1 y}=F_{1} \exp \left[i \int q_{1}(z) d z\right]+F_{2} \exp \left[i \int q_{2}(z) d z\right]$,

where $F_{1}$ and $F_{2}$ are constants that must be determined from the boudary conditions. The equation for $q_{1,2}$ reads

$q^{2}-\frac{i A}{\eta_{m}} q-\frac{D}{\eta_{m}}-i q^{\prime}=0$.

If the wavelength is small, the last term on the 1.h.s. is also small compared to the first one and it can be considered as a perturbation. Therefore, Eq.(16) can be solved by making use of the standard perturbation procedure. If we represent the quantity $q$ as a sum of subsequent perturbation terms, $q=q^{(0)}+q^{(1)}+\ldots$, then the first two terms in this expansion satisfy the following equation

$q^{(0) 2}-\frac{i A}{\eta_{m}} q^{(0)}-\frac{D}{\eta_{m}}=0, \quad q^{(1)}=\frac{i q^{(0)}}{2 q^{(0)}-i A / \eta_{m}}$.

These two terms are sufficient for our consideration. It follows from Eq.(17) that there are two thermomagnetic modes and $q^{(0)}$ for these modes is given by 
$q_{1,2}^{(0)}=\frac{i A}{2 \eta_{m}} \pm \sqrt{\frac{D}{\eta_{m}}-\frac{A^{2}}{4 \eta_{m}^{2}}}$.

Estimating different terms in $q_{1}$ and $q_{2}$, one has $A \sim c_{e}^{2} \tau_{e} / L$ and $D \sim c_{e}^{2} \tau_{e} / L^{2}$ where $L$ is the vertical lengthscale. Since $\left(D / \eta_{m}\right) /\left(A^{2} / 4 \eta_{m}^{2}\right) \ll 1$, we have

$\sqrt{\frac{D}{\eta_{m}}-\frac{A^{2}}{4 \eta_{m}^{2}}} \approx \frac{i A}{2 \eta_{m}}\left(1-\frac{2 \eta_{m} D}{A^{2}}\right)$.

Substituting this expression into Eq. (18), we obtain

$q_{1}^{(0)} \approx \frac{i A}{\eta_{m}}-\frac{i D}{A}, \quad q_{2}^{(0)} \approx \frac{i D}{A}$.

As it was noted, $\gamma, F_{1}$, and $F_{2}$ should be determined from the boundary conditions. Generally, the results depend on boundary conditions but this dependence is not very essential for short wavelength modes. Therefore, we consider the simplest case assuming that $B_{1 y}$ is vanishing at the bottom $\left(\left(B_{1 y}=0\right.\right.$ at $\left.z=0\right)$ and the electric current is zero at the top $\left(d B_{1 y} / d z=0\right.$ at $\left.z=a\right)$. This choice seems to be plausible because the thermal generation cannot operate in deep layers where the rates of thermomagnetic processes becomes very low. Also, the considered mechanism generates only the toroidal magnetic field $B_{1 y}$ and, hence, the electric current should vanish at the stellar surface. From the condition $B_{1 y}=0$ at $z=0$, we obtain $F_{1}=-F_{2}$, and then

$B_{1 y}=F_{1}\left\{\exp \left[i \int_{0}^{z} q_{1}(z) d z\right]-\exp \left[i \int_{0}^{z} q_{2}(z) d z\right]\right\}$.

The second boundary condition $\left(d B_{1 y} / d z=0\right.$ at $\left.z=a\right)$ yields

$q_{1}(a)-q_{2}(a) \exp \left[i \int_{0}^{a}\left[q_{2}(z)-q_{1}(z)\right] d z\right]=0$,

By making use of Eq. (20), we obtain

$q_{1}(a)-q_{2}(a) \exp \left[\int_{0}^{a}\left(A / \eta_{m}\right) d z\right]=0$.

The solution of this equation is crucially dependent on the sign of $A$ that is determined by $d T / d z$. If $d T / d z<0$ and the temperature decreases outward then the exponential term on the 1.h.s. of Eq.(23) is small since $\left|\int_{0}^{a}\left(A / \eta_{m}\right) d z\right| \sim\left|a A / \eta_{m}\right| \sim(a / L) \varepsilon$ is large and negative in the region where $\varepsilon \gg 1$ and termomagnetic effects are important. Therefore, one can neglect the exponential term in Eq. (23) and the dispertion relation reads $q_{1}(a) \approx 0$ in this case. Then, using Eq. (20), one has

$\frac{A}{\eta_{m}}-\frac{D}{A} \approx 0$

or, using Eq.(18),

$\gamma \approx-\frac{A^{2}}{\eta_{m}}+D_{0}$

Since $A^{2} / \eta_{m} \gg D_{0}$ (see Eq. (19) and the discussion above it), we obtain $\gamma \approx-A^{2} / \eta_{m}<0$ and, hence, generation of the magnetic field is impossible if $d T / d z<0$.

In the region with the inverse temperature gradient, $d T / d z>$ 0 , the situation differs drastically. In this case, $A$ is positive and the second term on the 1.h.s. of Eq. (23) gives a dominating contribution since the exponent is proportional to $\int_{0}^{a}\left(A / \eta_{m}\right) d z \sim a A / \eta_{m} \sim$ $(a / L) \varepsilon \gg 1$. Therefore, the first term can be neglected and the dispersion relation reads $q_{2}(a) \approx 0$ or $D(a)=0$. Then,

$\gamma \approx D_{0}(a) \approx 0.81 \frac{k_{B}}{m_{e}} \frac{d}{d z}\left(\tau_{e} \frac{d T}{d z}\right)-\eta_{m} k_{x}^{2}$
Taking into account that $\tau_{e} \propto T^{3 / 2} / n$ (Spitzer 1998), we obtain

$\gamma \sim 0.81 \frac{k_{B}}{m_{e}} \tau_{e}\left[\frac{3}{2 T}\left(\frac{d T}{d z}\right)^{2}-\frac{d \ln \rho}{d z} \frac{d T}{d z}+\frac{d^{2} T}{d z^{2}}\right]-\eta_{m} k_{x}^{2}$.

Generally, $\gamma$ can be positive or negative depending on the temperature and density profiles. It appears that $\gamma$ is positive in some layers with the inverse temperature gradient and the instability occurs in these layers. Such layers are formed in massive stars because of their high luminosity and departures from the local thermodynamic equilibrium in the upper atmosphere. This behaviour of $T$ is well known from the atmospheric models developed first by Auer \& Mihalas (1969a,b) and confirmed later by many authors (see, e.g., Gabler et al. 1989 and Martins 2004 for review). Because of a departure from the local thermodynamic equilibrium, the temperature profile in the atmosphere of massive stars has a bump-like structure in the region where the optical depth is less than 1 (see, e.g., Fig. 2.1 after Martins 2004). The bump in a temperature profile is typically located at the optical depth $\sim 0.01-0.001$ and its height depends on the surface temperature. In our model, we can assume that the bottom of a generating layer ( $z=0$ in our notations) corresponds to the depth where the temperature gradient changes the sign. Then, it is easy to check that $\gamma>0$, at least in a fraction of the bump region. Indeed, the first term in the brackets on the r.h.s. of Eq.(27) is always positive. The second term is also positive in layers with the inverse temperature profile $(d T / d z>0)$. The third term, $d^{2} T / d z^{2}$, should be positive in a some region with $d T / d z>0$ because of a bump-like shape of the temperature profile. Hence, there always exist the region where $\gamma>0$ and the instability occurs. The characteristic lengthscale of this region is comparable to the thickness of a layer with the inverse temperature gradient.

\section{DISCUSSION}

This paper considers the thermal generation of the magnetic field in surface layers of massive stars. Such generation is possible in the upper atmosphere of hot stars where departures from the local thermodynamic equilibrium form a region with the inverse temperature gradient. Such a behaviour of $T$ is well known from atmospheric modelling and is rather general in massive stars (see, e.g., Auer \& Mihalas 1969a,b, Martins 2004). An effectivity of the thermal generation is determined by value of the inverse temperature gragient and thickness of this layer. The inverse temperature gradient exists typically in layers with the optical depth $\tau<0.01-0.001$.

We have considered only generation of small-scale magnetic fields with the horizontal wavelengths $\lambda=2 \pi / k_{x}$ shorter than the lengthscale of unperturbed quantities, $L$. Note, however, that the considered mechanism can generate magnetic fields with the lengthscale comparable to $L$ as well. Certainly, unstable perturbations do not have a wavelike shape in this case. For instance, generation of such fields has been studied by Urpin et al. (1986) in neutron stars. It follows from Eq.(27) that, in a short-wavelength approximation $(L \gg \lambda), \gamma$ reaches its maximum if $k \rightarrow 0$ (or $\lambda \rightarrow \infty)$. However, Eq.(27) does not apply if $\lambda \geqslant L$ and the maximum lengthscale of unstable perturbations is restrickted by the lenghtscale of unperturbed quantities, $L$. Numerical calculations by Urpin et al. (1986) for $\lambda \geqslant L$ confirm this qualitative conclusion. Indeed, $\gamma \rightarrow 0$ if $\lambda \rightarrow \infty$ and the maximum value of the growth rate is reached at $\lambda \sim L$ and is given by Eq.(27) at $\lambda \sim L$.

The condition of instability for short-wavelenghts reads $\gamma>0$ and, hence, the minimum unstable wavelength can be estimated as $\lambda_{\min } \sim L \varepsilon^{-1 / 2}$. Therefore, the range of unstable wavelengths 
is $\lambda_{\min }<\lambda<L$, and these fields can manifest themselves as magnetic spots on the surface. Such spots are in qualitative agreement with the lengthscale of observed magnetic spots. Indeed, if $T=3 \times 10^{4}$, then $\varepsilon^{-1 / 2} \sim 30$ in the atmosphere where $n_{13}=1$. Since, $L \sim 10^{9} \mathrm{~cm}$ in massive stars, generated magnetic spots have a lengthscale $\simeq 3 \times 10^{10} \mathrm{~cm}$.

By making use of Eq.(27), the growth rate of magnetic disturbances can be estimated as

$\left.\gamma \sim\left(k_{B} / m_{e}\right) \tau_{e}(3 / 2 T)(d T / d z)\right)^{2} \sim c_{e}\left(\lambda_{e} / L^{2}\right)$,

where $\lambda_{e}=c_{e} \tau_{e}$ is the mean free-parth of electrons. The standard expression for the electron relaxation time (Spitzer 1998) yields the following estimate for the growth time $t_{B}=1 / \gamma$ of disturbances

$t_{B} \sim 10^{3} n_{13} L_{9}^{2} \Lambda T_{4}^{-5 / 2} \mathrm{yrs}$,

where $L_{9}=L / 10^{9} \mathrm{~cm}$. For typical parameters $\left(n_{13}=L_{9}=1\right.$, $\Lambda=4, T_{4}=3$ ), we obtain $t_{B} \sim 10^{4}-10^{5} \mathrm{yrs}$. The proposed mechanism cannot generate the magnetic field in very young stars (with the age $<10^{4} \mathrm{yrs}$ ). The generation is possible, however, for older stars. The main-sequence lifetime of massive stars, $\tau_{m s}$, is relatively short but, nevertheless, the timescale of the magnetic field generation can be shorter. The lifetime of massive stars is

$\tau_{m s} \approx 10^{10}\left(M / M_{\odot}\right)^{-2.5} \mathrm{yr}$,

where $M$ is the stellar mass (see, e.g., Bhattacharya \& van den Heuvel 1991, Urpin et al. 1998). The lifetime of very massive stars (with $M>100 M_{\odot}$ ) is obviously shorter than $t_{B}$ and, hence, the field cannot be generated in very massive stars. As far as less massive stars are concerned, generation by the thermal mechanism is possible for such stars.

Thermomagnetic waves have a simple form with vanishing disturbances of all quantities except $B_{1 y}$ only in a linear regime and, hence, the thermomagnetic instability leads to an exponential growth of the magnetic field only in this regime. Likely, the magnetic disturbances play a crucial role in settling down a saturation regime. The thermal generation is determined by the Nernst effect and caused by the term proportional to $\beta_{\wedge}$ in induction equation (3). This generating effect is suppressed if the magnetic field becomes so strong that the Hall parameters $x_{e} \omega_{B} \tau_{e} \sim 1$. Likely, this condition yields an estimate of the saturation regime of the thermomagnetic instability. Note that laboratory experiments and numerical modelling (see, e.g., Tidman \& Shanny (1974), Bol'shov, Dreizin, \& Dykhne (1974), and Andrushchenko \& Pavlenko (2004)) are in a qualitative agreement with this simple estimate of a saturation state. The condition $x_{e} \omega_{B} \tau_{e} \sim 1$ yields the following estimate for a saturation magnetic field

$B_{\text {sat }}=\frac{m_{e} c}{e \tau_{e}} \sim 10^{2} \frac{n_{13} \Lambda}{T_{4}^{3 / 2}} \quad \mathrm{G}$.

In stellar interiors, the ratio $n / T^{3 / 2}$ usually increases with the depth from the surface and, therefore, the saturation field is stronger in deep layers. Eq. (31) yields the magnetic field that is in a qualitative agreement with that observed in massive stars.

Acknowledgements. The author thanks the Russian Academy of Sciences for financial support under the programme OFN-15.
Auer, L.H., \& Mihalas, D. 1969b. ApJ, 156, 681

Bhattacharya, D. \& van den Heuvel, E. 1991. Phys. Rep., 203, 1

Biermann, L. 1950. Naturforschg, 5, 65

Bissell, J.J., Ridgers, C.P, \& Kingham, R.J. 2010. Phys. Rev. Lett., 105, 175001

Bissell, J.J., Kingham, R.J., \& Ridgers, C.P. 2012. Phys. of Plasma, 19, 052107

Bissell, J.J. 2015. J. Plasma Phys., 81, 905810108

Bol'shov, L.A., Dreizin, Y.A., \& Dykhne, A.M. 1974. JETP Letters, 19,168

Braginskii, S. 1965. In "Reviews of Plasma Physics" (Ed. M.Leontovich), vol. 1, p. 205, Consultants Bureau, New York

Brandenburg, A. \& Urpin, V. 1998. A\&A, 332, L41

Brownell, J. 1979. Comm. Plasma Phys. \& Control. Fus. 4, 131

Cantiello, M., Braitwaite, J. 2011. A\&A 534, A140

Doi, K., Susa, H. 2011. ApJ, 741, 93

Dolginov, A., Urpin, V. 1979. ZhETF, 77, 1921

Gabler, R., Gabler, A., Kudrizki, R.P., Puls, J., \& Pauldrach, A. 1989. A\&A, 226, 162

Franko, A., Montani, G., \& Carlevaro, N. 2014. PhysD, 288, 23

Haines, M. 1981. PhRvL, 47, 917

Hirao, A. \& Ogasawara, M. 1981. J. Phys. Soc. of Japan, 50, 668

Hubrig, S., Schoeller, M., Kholtygin, A., Oskinova, L., Ilyin, I. 2016. astro-ph/1602.08930

Kemp, J.C. 1982. PASP, 94, 627

Kholtygin. A., Hubrig, S., Valyavin, G., Fabrika, S., Chuntonov, G., Dushin, V., \& Milanova, Y. 2015. Astron. Soc. Pac. (Conf. Ser.), 494, 221

Martins, F. 2004. PhD Thesis, University Paul Sabaier, Toulouse Mestel, L. \& Moss, D. 1983. MNRAS, 204, 557

Montani, G., Benini, R., Carlevaro, N., \& Franko, A. 2013. MNRAS, 436, 327

Ojha, S.N. 1987. ApSS, 135, 175

Sana, H., de Mink, S., de Koter, A., Langer, N., Evans, C., Gieles, M., Gosset, E., Izzard, R., Le Bouquin, J., \& Schneider, F. 2012. Science, 337, 444

Schöller et al. 2016, astro-ph/1611.04502

Spitzer L. 1998. Physical Processes in the Interstellar Medium. Wiley-VCH

Tidman, D., Shanny, R. 1974. Phys. Fluids, 17, 1207

Urpin, V. 1981. Ap\&SS, 79, 11

Urpin, V., Konenkov, D., \& Geppert, U. 1998. MNRAS, 299, 73

Urpin, V., Levshakov, S.,\& Yakovlev, D.1986. MNRAS, 219, 703

Urpin, V., \& van Riper, K. 1993. ApJ, 411, L87

Urpin, V., Chanmugam, G., \& Sang, Y. 1994. ApJ, 433, 780

Urpin, V., \& Rüdiger, G. 2005. A\&A, 437, 23

Yoshizawa, A. 1990. PhFlB, 2, 1589

Wang, K.C. 1966. Phys. Fluids, 9, 1922

Wickramasinghe, D.T., Tout, C., \& Ferrario, L. 2014. MNRAS, 437, 675

\section{REFERENCES}

Ando, M., Doi, K., \& Susa, H. 2010. AIPC, 1238, 133

Andrushchenko, Zh., Pavlenko V. 2004. PhPl, 11, 1402

Auer, L.H., \& Mihalas, D. 1969a. ApJ, 156, 157 